\title{
Estado cognitivo del paciente de edad avanzada en programa de hemodiálisis
}

\section{David Gil Nieto ${ }^{1-}$ Montse Perera Díaz ${ }^{2}$ - Lidia Sevane Fernández ${ }^{1}$}

Servicio de Nefrología, Fundación Puigvert. Clinica Emilio Rotellar. Barcelona

${ }^{1}$ Fundación Puigvert - ${ }^{2}$ Clínica Emilio Rotellar

\section{Resumen}

El envejecimiento de la población en tratamiento renal sustitutivo, su estilo de vida, así como su pluripatología van asociados a trastornos cognitivos. Este deterioro cognitivo se puede identificar y cuantificar con instrumentos como el Mini Mental State Studio.

Nuestro objetivo fue evaluar el estado cognitivo de una muestra de pacientes de edad avanzada en programa de hemodiálisis y compararlo con el de la población general. Para ello se estudió en 46 pacientes mayores de 70 años en programa de hemodiálisis en la Fundación Puigvert y en la Clínica Emilio Rotellar en 2007. Se tuvieron en cuenta variables correspondientes a edad, diabetes, hipertensión y nivel de estudios. Se aplicó el test Mini-Mental Satate Examinatios en su versión ampliada a 37 items.

Los resultados muestran que la media de edad fue de 77 años, $83 \%$ hipertensos, 11\% diabéticos, con una media en hemodiálisis de 4,5 años y con un mayor nivel de estudios. Presentaban un probable deterioro cognitivo mayor que el de la población general, lo que es una fuente de dificultad, por lo que debe medirse y tenerse en consideración a la hora de realizar la planificación de una intervención educativa.

Correspondencia:

Lidia Sevane Fernández

Av. Madrid, 5. Escalera B, 5०3

08028 Barcelona

lidia_sf@hotmail.com
PALABRAS CLAVE:

- HEMODIÁLISIS

- DETERIORO COGNITIVO

- ENVEJECIMIENTO

\section{Cognitive state of elderly patient on haemo- dialysis programme}

\section{Abstract}

The aging of the population receiving renal replacement therapy, their lifestyle, and their pluripathology are associated with cognitive disorders. This cognitive impairment can be identified and quantified using instruments such as the Mini Mental State Examinations.

Our aim was to evaluate the cognitive state of a sample of elderly patients on haemodialysis and compare it to that of the general population. To do so we studied 46 patients aged over 70 on the haemodialysis programme at the Fundación Puigvert and at the Clínica Emilio Rotellar in 2007. Variables relating to age, diabetes, hypertension and level of studies were taken into account. The Mini-Mental State Examinations test was applied, using the version extended to 37 items.

The results showed that the average age was 77, $83 \%$ had hypertension, $11 \%$ were diabetic, with an average time on haemodialysis of 4.5 years and with higher education. These patients showed a greater likelihood of cognitive impairment than the general population, which is a source of difficulty, and should therefore be measured and taken into consideration when planning an educational intervention. 


\section{KEY WORDS:}

- HAEMODIALYSIS

- COGNITIVE IMPAIRMENT

AGING

\section{Introducción}

El envejecimiento de la población en tratamiento renal sustitutivo, así como su pluripatología, marcan nuevos retos a los profesionales sanitarios. A los años van asociados los trastornos cognitivos influenciados por el estilo de vida, factores de riesgo cardiovascular y niveles elevados de ácido úrico ${ }^{1,2}$. El deterioro cognitivo es uno de los principales problemas de los ancianos, con repercusión sobre ellos mismos, sus cuidadores formales e informales y sobre el sistema de salud $^{3}$. La prevalencia de demencia en mayores de 65 años se sitúa en un $10 \%$ y aumenta conforme avanza la edad en la población. La frecuencia de la alteración cognitiva moderada-severa presenta mayor variabilidad cifrándose entre el $6-42 \% \%^{4,5}$.

El deterioro cognitivo de la persona, ya sea primario o secundario a otra patología, se puede identificar y cuantificar con instrumentos de medida como el Mini Mental State Studio (MMSE) $)^{6-8}$ que contrastado y validado se usa en estudios epidemiológicos internacionales y en la práctica clínica para objetivar la presencia de déficit cognitivo. Su sensibilidad es del $87-100 \%$ y su especificidad del $62-100 \%$ en función del punto de corte para el cribado en el diagnostico de demencia 9-12. EI MMSE puede ser aplicado sin necesidad de ser especialista, no estando limitado en el tiempo aunque no precisa más de 10 minutos ${ }^{13}$. Existe una versión diseñada por un grupo de la Organización Mundial de la Salud que incluye los datos normativos y las instrucciones de aplicación ${ }^{14,15}$. Se han definido los parámetros del rendimiento cognitivo de una muestra poblacional española de ancianos mayores de 70 años mediante varias pruebas de utilización frecuente en la clínica y la investigación, incluyendo la aplicación del MMSE ${ }^{15}$.

Un deterioro cognitivo leve-moderado puede generar dificultades de adaptación a la situación derivada de la enfermedad renal. Las herramientas de valoración neurológica son un elemento clave para evaluar el grado del deterioro cognitivo y su repercusión en el manejo de la enfermedad ${ }^{16}$. Algunos de los diagnósticos más frecuentes en los pacientes en programa de hemodiálisis (HD) son el manejo inefectivo del régimen terapéutico, el desequilibrio nutricional por defecto y los conocimientos deficientes, recogidos en la guía de diagnósticos del paciente en hemodiálisis ${ }^{17}$. La educación para la salud es la intervención común a todos ellos dirigida al enfermo y al cuidador, como parte integral del rol enfermero. Es habitual la detección de un déficit de conocimientos y la eficacia de la intervención educativa ${ }^{18}$. El personal de enfermería dado su intenso contacto con el paciente y su entorno se sitúa en una posición privilegiada para obtener una valoración real de los conocimientos y actitudes de las personas objetivo de sus cuidados. Los enfermos que acuden a consulta prediálisis, gracias a las acciones educativas que se llevan a cabo, muestran mayor conocimiento de su enfermedad y todo lo relacionado con ella ${ }^{19}$. Además, la educación paciente/familia reduce el coste causado por las complicaciones de la enfermedad. Es importante dar una información adecuándola en cantidad y calidad a las capacidades y actitudes del individuo, encaminada a crear y potenciar hábitos saludables.

\section{Objetivo}

Evaluar el estado cognitivo de una muestra de pacientes de edad avanzada en programa de hemodiálisis y compararlo con el de la población general.

\section{Material y Método}

Estudio cuantitativo, descriptivo y comparativo. Se seleccionó una muestra de pacientes mayores de 70 años con IRC en tratamiento sustitutivo de hemodiálisis. Como criterio de inclusión se consideró a los pacientes mayores de 70 años de edad en programa de HD crónica en la Fundación Puigvert y la Clínica Emilio Rotellar, durante el segundo semestre del 2007. Quedan excluidos aquellos que presentan deterioro cognitivo grave (con o sin diagnóstico), algún déficit sensorial o motor o enfermedad mental (con o sin diagnóstico) por imposibilidad de responder a algunas cuestiones del MMSE.

La muestra incluyó un total de 46 sujetos. La mitad correspondían al total de la población mayor de 70 
años en programa de HD en la Fundación Puigvert. De esta mitad se descartaron 4 individuos (1 por presentar deterioro cognitivo grave, 2 por limitaciones sensoriales y otro por enfermedad mental). En Clínica Emilio Rotellar en el momento de la selección recibían tratamiento 96 pacientes mayores de 70 años de los que a priori se descartaron 4 (1 por presentar limitación sensorial, 2 por deterioro cognitivo grave y otro por enfermedad mental). Del resto se seleccionaron 25 sujetos por su mayor disponibilidad para realiza el test MMSE sin alterar la dinámica de funcionamiento de las unidades. Todas las pruebas se realizaron pre-HD.

Se registraron variables correspondientes a edad, diabetes, hipertensión y nivel de estudios. Se aplicó el test Mini-Mental State Examination (MMSE) en su versión ampliada a 37 ítems, diseñada por un Grupo de la Organización Mundial de la Salud para la aplicación de estudios epidemiológicos ${ }^{14}$. Se consideró sin estudios aquellos sujetos que realizaron menos de 4 años de estudios reglados, variable a considerar por su incidencia demostrada en la puntuación del test aplicado $6,9,10$.

El tratamiento de datos se realizó mediante el programa estadístico SPSS 16.0. Los datos estandarizados del rendimiento cognitivo de una muestra poblacional española son los publicados anteriormente por Ser Quijano, et al. ${ }^{15}$. Las variables cualitativas se expresan como valor absoluto y en porcentaje. Las variables cuantitativas se expresan como media y desviación típica. La comparación de las medias se realizó mediante la $\mathrm{t}$ de Student para muestras independientes en variables cuantitativas y $\mathrm{X}^{2}$ para el nivel de estudios. Se consideró estadísticamente significativo valores de $p<0,05$.

\section{Resultados}

Los 46 pacientes en HD estudiados presentaban una media de 77,48 $\pm 4,74$ años de edad. El 82,6\% eran hipertensos y el $10,86 \%$ diabéticos. Con una media de 4,55 $\pm 4,98$ años en tratamiento sustitutivo renal. No existieron diferencias significativas respecto a la variable edad entre la muestra poblacional y la de pacientes en HD ( $p=0.387)$. La proporción por estratos de edad en ambas muestras queda reflejada en la tabla 1 y la figura 1 .

\begin{tabular}{|c|c|c|c|c|}
\hline & $\begin{array}{c}\text { Población } \\
\text { General }\end{array}$ & $\begin{array}{l}\text { Muestra } \\
\text { en Diálisis }\end{array}$ & $\begin{array}{l}\text { IC } \\
95 \%\end{array}$ & $\mathbf{P}$ \\
\hline Media edad & $\begin{array}{c}78,1[5,4] \\
(n=368)\end{array}$ & $\begin{array}{c}77,4[4,8] \\
n=46\end{array}$ & $\begin{array}{l}-0,2 \\
0,97\end{array}$ & 0.378 \\
\hline Hombres & 186 & 33 & - & - \\
\hline Mujeres & 182 & 13 & - & - \\
\hline $\begin{array}{l}<4 \text { años } \\
\text { de Estudios } \\
\text { Regulados }\end{array}$ & 246 & 14 & - & $<0,001$ \\
\hline $\begin{array}{l}>4 \text { años } \\
\text { de Estudios } \\
\text { Regulados }\end{array}$ & 104 & 32 & - & $<0,001$ \\
\hline Media MMSS & $\begin{array}{c}30(4,7) \\
n=368\end{array}$ & $\begin{array}{c}26,9(6,14) \\
n=46\end{array}$ & $\begin{array}{l}-4,46 \\
-1,23\end{array}$ & 0,002 \\
\hline \multicolumn{5}{|c|}{$\begin{array}{l}\text { P: Significancia estadística } \\
\text { [D]t: Desviación Típica } \\
\text { MMSS: Minimental } \\
\text { IC: Intervalo de Confianza }\end{array}$} \\
\hline
\end{tabular}

Tabla 1. Comparación de la muestra poblacional y de la muestra en hemodiálisis
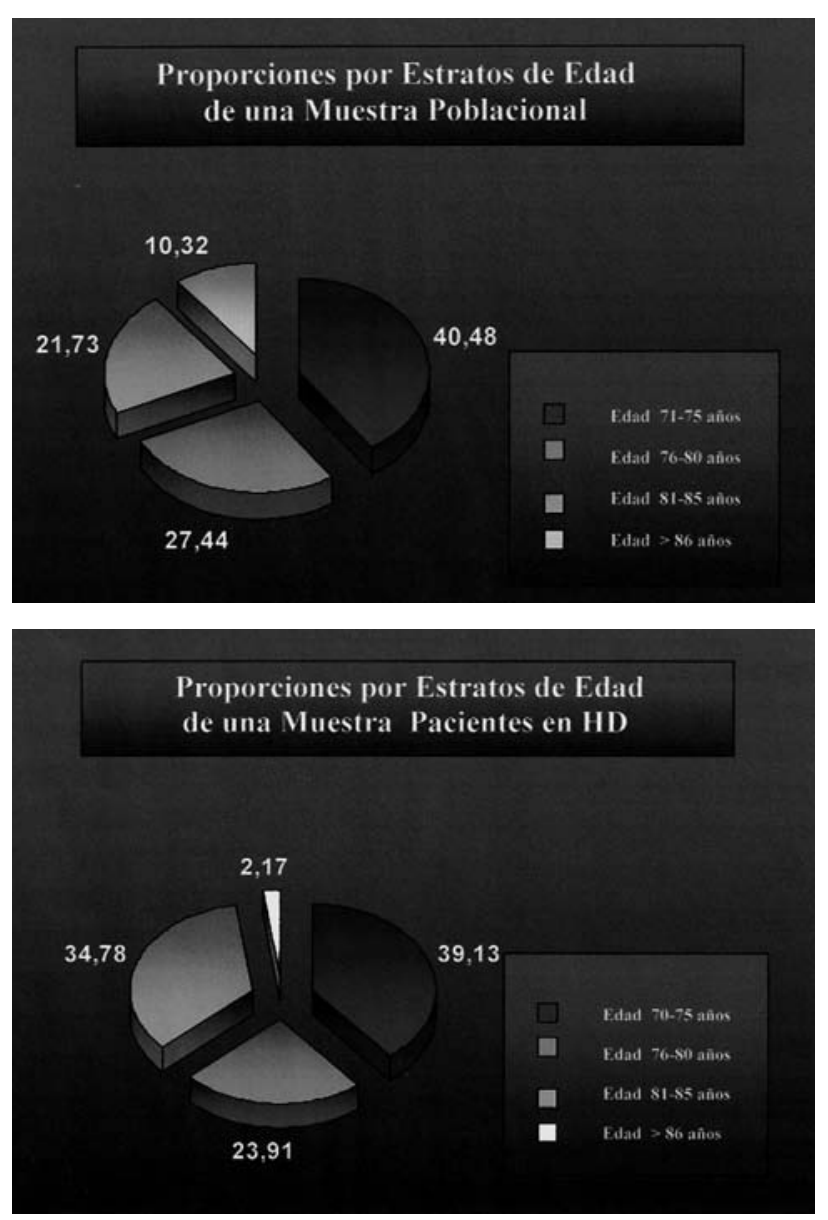

Figura 1. Proporción por estratos de edad en ambas muestras 
Existieron diferencias respecto al nivel de estudios $(p<0,001)$. El $69,56 \%$ de la muestra de pacientes en HD refirieron tener más de 4 años de estudios reglados, mientras que la proporción en la muestra poblacional era de un $28,26 \%$ (figura 2 ).
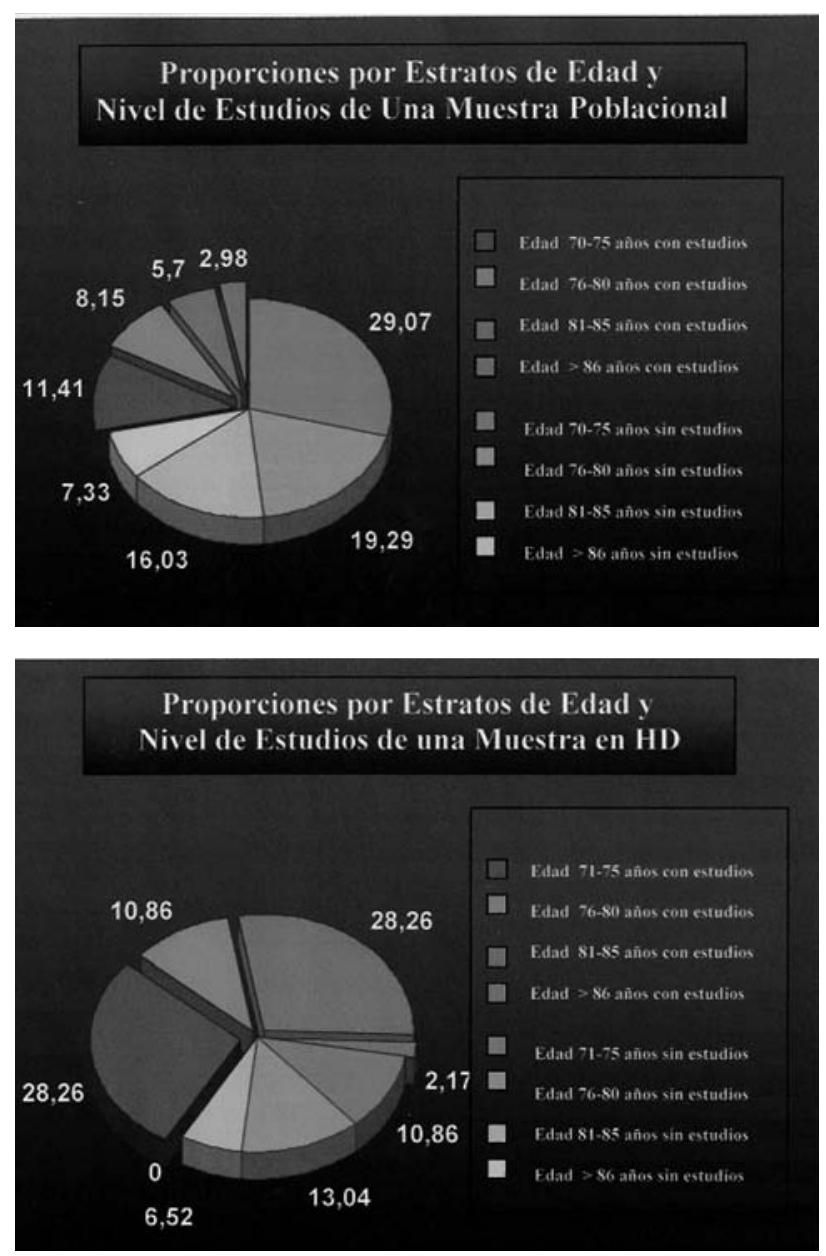

Figura 2. Proporción por nivel de estudios en ambas muestras

La calificación media en el MMSE en pacientes en hemodiálisis fue de $26,96 \pm 6,14$ puntos frente a una media de $30 \pm 4,7$ puntos de la media de la población general, siendo esta diferencia estadísticamente significativa $(p=0.002)$, con un intervalo de confianza del $95 \%$ de $-4,86$ a $-1,23$ (tabla 1 ). Los resultados de las dos muestras correspondientes a los centiles $-1,-1,5$ y -2 están representados en la figura 3.

Resulta no significativa ( $p=0.682$ ) la correlación de Pearson entre el resultado del MMSE y el tiempo de tratamiento.

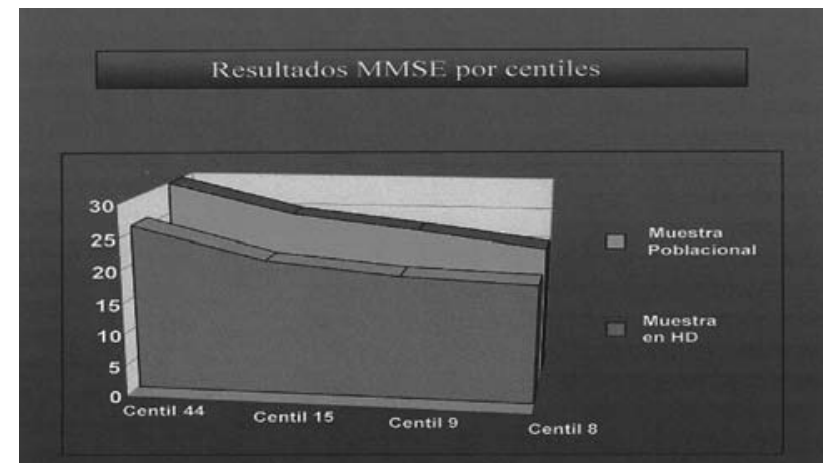

Figura 3.

\section{Discusión}

Tenemos una muestra en hemodiálisis de igual edad y con un mayor nivel de estudios que la muestra poblacional por lo que podríamos esperar un resultado igual o superior en el MMSE. Por el contrario obtenemos un resultado menor, siendo esta diferencia estadísticamente significativa. En la bibliografía encontramos que la edad es el factor más influyente en la presencia del deterioro cognitivo junto con el nivel estudios $^{1,5,10}$. Además, las alteraciones vasculares y patologías crónicas son también factores de riesgo, todos ellos presentes en la insuficiencia renal. Por tanto, no es paradójico obtener un menor resultado en un test que mide precisamente la esfera cognitiva de una muestra de pacientes de edad añosa en tratamiento sustitutivo de hemodiálisis respecto al resultado obtenido por Quijano en la población general. Si la muestra fuera mayor en número posiblemente podríamos obtener resultados significativos al realizar una correlación entre el tiempo en hemodiálisis y la puntuación obtenida en el MMSE.

En la práctica diaria de enfermería en las unidades de diálisis se observan cómo personas en tratamiento sustitutivo renal realizan elecciones de la vida diaria ineficaces para cumplir los objetivos del tratamiento, e incluso se verbalizan las dificultades de integrar y regular algunos de los regímenes terapéuticos prescritos. Estas manifestaciones, fuentes de dificultad por falta de fuerza, voluntad y conocimientos, generan un diagnóstico de manejo inefectivo del régimen terapéutico cuyas intervención NIC es la enseñanza, con indicadores NOC sobre dieta, proceso de enfermedad, medicación, actividad prescrita, evitar conductas que potencien la enfermedad, alteraciones 
funciones del rol para cumplir requisitos terapéuticos y procedimiento en el dominio de la prevención de la salud. La enseñanza es la intervención en el afrontamiento inefectivo, correspondiente al dominio de la prevención de la salud, en el desequilibrio nutricional, dominio de nutrición, y conocimientos deficientes, dominio de percepción-cognición.

Las conductas no adaptativas pueden acabar generando, entre muchos otros, disfunciones del acceso vascular o sesiones de hemodiálisis extras. Conductas que repercuten negativamente en el individuo y en el sistema sanitario. En el desempeño del rol enfermero, se realizan intervenciones dirigidas a la educación sanitaria. En las actividades para llevar a cabo una intervención es donde se evidencia el deterioro cognitivo de los enfermos más añosos. Un deterioro que dificulta la comprensión, reflexión e integración de conocimientos necesarios para desarrollar conductas adaptativas a la nueva situación.

Existe constancia de la relación entre un menor grado de comprensión verbal y la carencia de conocimientos después de una intervención de educación sanitaria ${ }^{19}$. Destaca también la importancia de una información adecuada al inicio y durante el tratamiento, ya que por si solos los cuidadores no aumentan el nivel de conocimientos $^{18}$. La eficacia de la intervención Enseñanza: grupo, mejora tanto a nivel individual como colectivo incrementando los cuidados percibidos por la persona ${ }^{20}$. Conocemos la existencia de posible deterioro cognitivo que dificulta la comprensión y transmisión de información desde los enfermos a sus cuidadores en aquellas tareas de suplencia fuera de las unidades de diálisis.

Constatada esta realidad cobran relieve las intervenciones realizadas sobre familiares y cuidadores informales. El abordaje no puede realizarse por otros canales de comunicación diferentes al trato personal. Quedan relegadas al seguimiento y evaluación las unidades de tratamiento por limitaciones estructurales y de dinámica de trabajo. El lugar idóneo para la actividad, donde se puede garantizar la máxima eficiencia y eficacia es la consulta de enfermería. En la primera fase del proceso de atención se podría incluir una herramienta de medida de la esfera cognitiva, utilizando este elemento como sistema de cribaje entre la intervención educativa al cliente que puede incluir a su entorno o la intervención al cliente y su entorno. El seguimiento y evaluación desde la unidad de diálisis debería utilizar un canal de información retroalimentado con la consulta de enfermería.

\section{Conclusiones}

La muestra de enfermos en hemodiálisis presenta un probable deterioro cognitivo mayor que el de la población general. Este deterioro es fuente de dificultad y debe medirse y tenerse en consideración a la hora de realizar la planificación de una intervención una intervención educativa.

\section{Agradecimientos}

A Amaranta Fuenmayor por dedicarnos su tiempo e inestimable ayuda. Sin ella estaríamos a oscuras en el desarrollo de este artículo. Al Dr. I. Gich por su asesoramiento estadístico.

\section{Bibliografía}

1. Benítez del Rosario, M.A. La trascendencia de los estudios epidemiológicos del deterioro cognitivo. Aten Primaria. 2003; 32 (1): 6-14.

2. Schretlen D.J, Inscore A.B, Jinnah H.A, Rao V, Goedon B, Pearlson G.D. Sermen úric ácid and cognitivo in Community-Dwelling Older aldults. Neuropsycology. 2007; Jan 21 (1): 136-140.

3. Limón Ramírez E, Argimón Pallàs J.M. Demencia, deterioro cognitivo leve y valores poblacionales de referencia. Med Clin (Barc). 2004; 122 (19): 744-5.

4. Martínez de la Iglesia J, Dueñas Herrero R, Onís Vilches M.C, Aguado Taberna C, Albert Colomer C, Luque Luque R. Adaptación y validación al castellano del cuestionario de Pfeiffer (SPMSQ) para detectar la existencia de deterioro cognitivo en personas mayores de 65 años. Med Clínica. 2001; 117(4): 129-134.

5. Martínez de la Iglesia J, Rubio Cuadrado M.V, Espejo Espejo J, Aranda Lara J.M, Enciso Berge I, Férula de Torres L.A, Francesca del Pozo F.J. 
Factores asociados a la alteración cognitiva en la población urbana. Proyecto ANCO. Aten Primaria. 1997; 2(7): 345-353.

6. Limón Ramírez E, Argión Pallás J, Vila Doménech J, Abos puez T, Cabezas Peña C, Vinyoles Bargalló E. Detección del deterioro cognitivo en población mayor de 64 años: primera fase del proyecto Cuidàl. Aten Primaria. 2003; 32(1): 6-14.

7. Benítez del Rosario M.A. Diagnóstico del deterioro cognitivo: dificultades metodológicas en la frontera de la normalidad cognitiva. Aten Primaria 2002; 30 (1): 5-15.

8. Vinyoles Bargalló E, Vila Doménech J, Argimón, Pallàs J, Espinós Boquet J, Albos Pueyop T, Limón Ramírez E. Concordancia entre el Mini-Examen Cognoscitivo y el Mini-Mental State Examination en el cribado del déficit cognitivo. Aten Primaria 2002; 30(1): 5-15.

9. González Moneo M.J, Escriva Ferreiro R, Vinyols Bargalló E, Espel Masferrer C, Davins Miralls J, Borrell Muñoz M. Estimaciones de la frecuencia del déficit cognitivo según el test empleado. Aten Primaria. 1997; 20(4): 173-179.

10. Pi J, Olivé J.M, Esteban M. Mini Mental State examination: asociación de la puntuación obtenida con la edad y grado de alfabetización en la población anciana. Med Clin (Barc). 1994; 103: 641644.

11. Escudero Sánchez C, García Cardona R, Ibáñez Colas A, López López M.A, Hidalgo García Consuegra M.A, Pérez Juárez $A$, Salan García $M$, Utrilla Bermejo F. Déficit cognitivo, prevalencia y factores asociados en la población mayor de 74 años. Aten Primaria. 1999; 24(6): 326-331.

12. Lobo A, Saz P, Marcos G, Díaz J.L, de la Camara C, Ventura T, Morales Asín F, Fernando Pascual L, Montañes J.A, Aznar S. Revalidación y normalización del Mini-Examen Cognoscitivo (primera versión en castellano del Mini-Mental Status Examination) en la población general geriátrica. Med Clin (Barc). 1999; 112; 767-774.
13. Herrero Botín M, Pascual Pérez A, Fernández Camino C. Valoración del estado cognitivo en los ancianos: Uso y significado del Mini-Mental State test. Enfermería científica. Num 116. 1991; 2629.

14. Bermejo Pareja F, Díaz Guzman J, Porta-Etessom J. Cien escalas de interés en neurología clínica. Barcelona: Proas Science. 2001; p. 85-7.

15. Ser Quijano T, García M.J, Sánchez F, Frades B, Bartolomé M.P, Otero A. Evaluación cognitiva del anciano. Datos normativos de la muestra poblacional española de más de 70 años. Med Clin (Barc) 2004; 122 (19) 727-40.

16. Beneges Búster, Blanca Cecilia. La valoración neurológica: un soporte fundamental para el cuidado de enfermería. Aquichón. Año 2000; (2): 4043.

17. Solcer Saer E, Campo Romero M.C, Carmona Valiente M.C, Fernández García J.L, Fernández Montero J.J, Ramírez Rodríguez. Guía de Diagnósticos de Enfermería del paciente en hemodiálisis. Grupo 2 Comunicación Medica. p. 13-42.

18. Bañabre González A, Vázquez Rivera J, Ontoriño Novoa S, Rodríguez González M, González Pascual M, Graña Álvarez J, Manso Feijó B, Otero López A. Efectividad de la intervención educativa en cuidados de pacientes dependientes en diálisis y valoración de la carga. Rev Soc Esp Enferm. Nefrol 2005; 8(2): 156-165.

19. Pérez Doctor R, Márquez Catalán D.M, Mejias Calero J.J. Valoración de la comprensión verbal y el conocimiento de la enfermedad renal en pacientes de diálisis. HYGIA 49: 27-30.

20. Gutiérrez Vilaplana $J M^{a}$, Samsó Piñol E, Cosi Ponsa J, Ibars y Moncasi P, Craver Hospital L. Evaluación de la intervención enseñanza: grupo en la consulta de enfermedad renal crónica avanzada. Rev. Soc. Esp. Enferm. Nefrol. 2007; 10(4): 280285. 